Universitas, Volumen 1, Número 2, 2008, 8-18

๑ 2008 UNAN-León, Editorial Universitaria

\title{
Metrología Química II: Estimación de la Incertidumbre en la Medición de pH en Aguas
}

\author{
Marco Delgado, Manuel Vanegas y Gustavo Delgado*.
}

Facultad de Ciencias y Tecnología, Departamento de Química, Laboratorio de Análisis de Trazas de Metales Pesados (LATMP), Edificio de Ciencias Básicas, León, Nicaragua. Tel: 505311 5013, ext: 1132. Fax: 505311 4012. Universidad Nacional Autónoma de Nicaragua, León (UNAN-LEÓN).

RESUMEN

El objetivo principal del presente trabajo es estimar la incertidumbre en la determinación del pH en una muestra de agua tomando en cuenta todos los parámetros experimentales que intervienen en el proceso de medición. Para evaluar la incertidumbre se siguió el procedimiento general establecido en la guía GUM ISO 1995. Se derivó el modelo matemático, donde interviene el efecto del potencial de asimetría del electrodo de vidrio utilizado. Se identificaron todas las fuentes de incertidumbres a través del diagrama causa-efecto. Se calcularon todos los componentes de las incertidumbres y se combinaron a través de la ley de propagación de la incertidumbre para obtener el valor de la incertidumbre estándar en la medición del pH en la muestra de agua $(u p H x)$. Se elaboró un diagrama de Pareto, donde se demuestra que de 7 fuentes principales de incertidumbre, solamente la repetibilidad en la lectura del potencial $(E x)$ de la muestra y la incertidumbre en el cálculo del $\mathrm{pH}$ donde el potencial es nulo $\left(\mathrm{pH}_{0}\right)$ son los que influyen en la estimación de $u p H x$, cuyo valor fue 0.05 . El intervalo de confianza encontrado fue de $7.6 \pm 0.11$. La incertidumbre expandida fue calculada tomando como factor de cobertura el valor de $t$ al $95 \%$ de nivel de confianza y 18 grados de libertad efectivos.

Palabras claves: Metrología química; Incertidumbre en la medición de pH en aguas; Incertidumbre expandida relativa en la medición de $\mathrm{pH}$; Potencial de asimetría.

\section{INTRODUCCIONN}

En la primera parte sobre la metrología química ${ }^{[1]}$ se estableció una metodología confiable para la calibración del sistema potenciométrico (pHmetro-electrodo) en las mediciones de $\mathrm{pH}$. Se evaluó la repetibilidad y se elaboraron las cartas de control para establecer los límites de control en las mediciones rutinarias de $\mathrm{pH}$. Son muchas las aplicaciones de este método potenciométrico para medir la acidez o basicidad en diferentes tipos de muestras, especialmente en análisis de agua residual y de consumo humano ${ }^{[2-4]}$.

Los intercambios comerciales exigen la certificación de la calidad de los productos siguiendo normas internacionales ${ }^{[5,6]}$. Las especificaciones de los productos deben reflejar la calidad de los resultados de la medición, lo que implica la exactitud y su grado de dispersión o incertidumbre asociada ${ }^{[7]}$. La metrología química desempeña un papel muy importante en el cálculo de las incertidumbres ${ }^{[8,9]}$. En las aguas de consumo humano es muy importante especificar el pH. Los certificados de este producto embotellado deben especificar su valor y la incertidumbre asociada.

Existen trabajos de investigación relacionados con la estimación de la incertidumbre ${ }^{[10]}$ y la trazabilidad ${ }^{[11]}$ en las mediciones de $\mathrm{pH}$. La mayoria de los resultados de los ensayos de aptitud o estudios de ínter comparación en estas mediciones arrojan grandes sesgos y muy poca precisión.
Por lo tanto, se hace necesario implementar un procedimiento normalizado para la calibración ${ }^{[1]}$ y la evaluación de la incertidumbre en las mediciones de $\mathrm{pH}$.

Existen diferentes guías generales ${ }^{[12-14]}$ que establecen las diferentes etapas para estimar la incertidumbre en las mediciones analíticas. Los procedimientos contemplan la derivación del modelo matemático, la cuantificación de los diferentes componentes de la incertidumbre, su combinación y la evaluación de la incertidumbre expandida.

El presente trabajo pretende establecer una metodología rigurosa para estimar la incertidumbre en la medición del pH en una muestra de agua, basado en la guía GUM ISO $1995^{[12]}$ para su expresión cuantitativa.

\section{TEORIA}

\section{Principio de la medición del pH}

El pH fue definido por Sorensen y Linderstrom-Lang en 1924 como el logaritmo negativo de la actividad del protón hidratado:

$$
p H=-\log a_{H}
$$

El electrodo primario para medir el $\mathrm{pH}$, es el de hidrógeno tomando como referencia un electrodo de plata-cloruro de plata.

\footnotetext{
*Autor para correspondencia: gusdel53@yahoo.es
} 
Este sistema sólo lo tienen los laboratorios de referencia nacionales, como el NIST en los Estados Unidos de América o el CENAM de México. Son los que fabrican soluciones de referencia primarias certificadas para $\mathrm{pH}$ con una incertidumbre de hasta 0.0005 unidades de $\mathrm{pH}^{[15]}$. Los laboratorios de ensayos utilizan materiales de referencia terciarios certificados trazables con NIST para calibrar el sistema potenciométrico, disponiendo de electrodos de vidrio comerciales.

Para medir el pH de una solución muestra con un electrodo de vidrio combinado con uno de plata-cloruro de plata como referencia, utilizando una solución de referencia certificada trazable con NIST $\left(\mathrm{pH}_{\mathrm{B}}, 7.00\right)$, se miden los potenciales de celda respectivos $\left(E_{X}\right.$ y $\left.E_{B}\right)$ a una temperatura dada y se calcula su $\mathrm{pHx}$ según el modelo de la ecuación (2) denominado $\mathrm{pH}$ operacional [16,17].

$$
p H_{X}=p H_{B}+\frac{\left(E_{B}-E_{X}\right) F}{2.303 R T}
$$

Donde R y F son las constantes de los gases y de Faraday con valores correspondientes a $8.31441 \mathrm{~J} \mathrm{~mol}^{-1} \mathrm{~K}^{-1} \mathrm{y}$ $96484.56 \mathrm{Cmol}^{-1[18]}$. T es la temperatura termodinámica. El potencial $E_{B}$ es diferente de $0.000 \mathrm{~V}$, debido a la existencia del potencial de asimetría provocado por las imperfecciones en la fabricación de las membranas de vidrio. Las mediciones de $\mathrm{pH}$ con dos o más soluciones de referencia tienen mayor precisión y exactitud ${ }^{[10,11]}$.

Estos modelos toman en cuenta la pendiente experimental y el coeficiente de variación de la pendiente como función de la temperatura. La medición de $\mathrm{pH}$ se realiza comúnmente utilizando electrodos de vidrio combinados comerciales de muy variada calidad ${ }^{[19]}$.
Estos electrodos se caracterizan por tener membranas de vidrio con capacidad de realizar mediciones en una amplia gama de $\mathrm{pH}$ que puede oscilar entre $1 \mathrm{y}$ 13. Fuera de este intervalo, los electrodos presentan respuestas erróneas. A pH menores que la unidad, la elevada concentración de protones hidratados bloquean los sitios activos y se tienen respuestas con errores negativos ${ }^{[2]}$.

Mientras que, a pH mayores que 13 los electrodos responden a iones alcalinos y se tienen errores positivos, debido a que el coeficiente de selectividad por el ion alcalino es grande ${ }^{[2,19-20]}$. Electrodos con membranas de vidrio de mala calidad pueden responder a una estrecha gama de $\mathrm{pH}$ que puede oscilar entre 2 y 9 . No obstante, dependiendo del alcance de las mediciones en el laboratorio, se podrá seleccionar el electrodo según se describe en el procedimiento del método normalizado.

\section{Procedimiento general para la estimación de la incertidumbre}

En cualquiera de los casos, se hace necesaria la evaluación de la incertidumbre en las mediciones de $\mathrm{pH}$, previa calibración del sistema potenciométrico tal a como se estableció anteriormente ${ }^{[1]}$. El procedimiento general para estimar la incertidumbre se presenta en la figura 1, de acuerdo a las directrices existentes para la estimación de la incertidumbre ${ }^{[12-14]}$.

En primer lugar se deriva el modelo matemático, se identifican las fuentes de incertidumbres (las de tipo A, obtenidas a partir de una serie de observaciones y

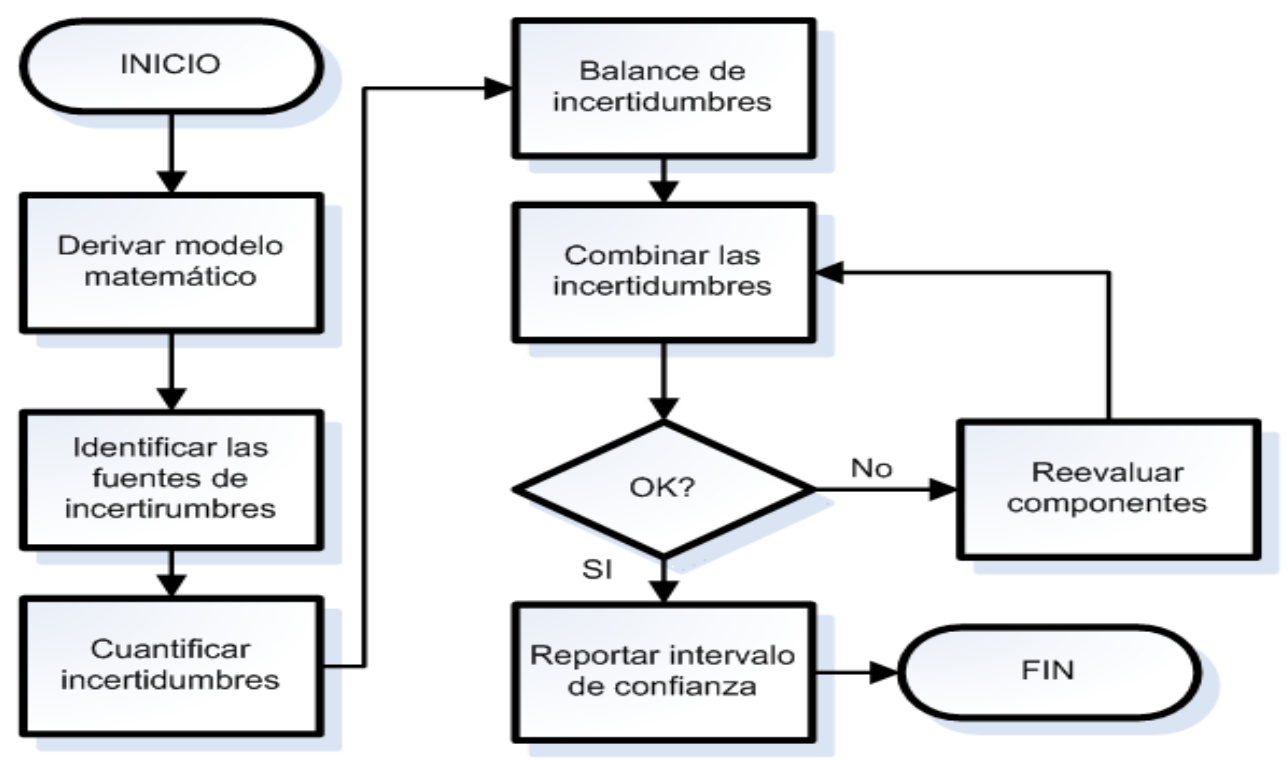

Figura 1: Diagrama de flujo representando el procedimiento para la estimación de la incertidumbre en la medición del pH. 
las de tipo $B$, obtenidas a partir de las distribuciones normal, rectangular $\mathrm{o}$ triangular), se cuantifican las incertidumbres, se hace un balance de las incertidumbres, se combinan las incertidumbres a través de la ley de la propagación de la incertidumbre, se revisa si no hay incongruencias para reevaluar los componentes de la incertidumbre, se calcula el intervalo de confianza especificando el factor de cobertura y finalmente se reportan los resultados con el número de cifras significativas correcto.

En el presente trabajo, se pretende aplicar este procedimiento para evaluar la incertidumbre en la medición de $\mathrm{pH}$ en una muestra de agua, derivando un modelo matemático que toma en cuenta el potencial de asimetría experimental del electrodo de vidrio.

\section{PARTE EXPERIMENTAL}

\section{Materiales}

Durante el proceso de calibración a 25 y $35^{\circ} \mathrm{C}$, se utilizaron soluciones de referencia de $\mathrm{pH} 4.00,7.00$ y 10.00 certificadas trazables con NIST, un pH-metro TACUSSEL LPH 230T (resolución $1 \mathrm{mV}$, precisión $\pm 1 \mathrm{mV}$, drift $<3 \mathrm{mV} / 12 \mathrm{~h}$, ajuste de temperatura con resolución de $0.1{ }^{\circ} \mathrm{C}$ ), un baño termostático Vidra FOC BT-9, una unidad refrigerante marca Vidra FOC UR-1, un termómetro digital TESTO 925 con resolución de $0.1^{\circ} \mathrm{C}$ y precisión $\pm 0.1^{\circ} \mathrm{C}$, un electrodo de vidrio combinado con el de referencia plata-cloruro de plata marca TACUSSEL $( \pm 0.02 \mathrm{pH})$.

\section{Calibraciones a 25 y $35^{\circ} \mathrm{C}$ y lectura del potencial de la muestra de agua}

El instrumental potenciométrico se calibró, de acuerdo, al procedimiento establecido en nuestro laboratorio en un trabajo anterior ${ }^{[1]}$. Se sumergió el electrodo de vidrio combinado en la solución de referencia de $\mathrm{pH} 7.00$ termostatizada a $25^{\circ} \mathrm{C}$ con una precisión de $\pm 0.1^{\circ} \mathrm{C}$, y se realizó la lectura en $\mathrm{mV}$.

Luego, se introdujo el electrodo en las soluciones de referencias de $\mathrm{pH} 4.00$ y 10.00 y se realizaron las lecturas correspondientes en $\mathrm{mV}$. Se hicieron 5 lecturas en milivoltios para cada valor de $\mathrm{pH}$. Este mismo procedimiento se utilizó para el proceso de calibración a $35^{\circ} \mathrm{C}$. Se midió la temperatura a la muestra con una precisión de $\pm 0.1^{\circ} \mathrm{C}$ y se introdujo el electrodo de vidrio, efectuándose 9 lecturas de potencial en milivoltios.

\section{RESULTADOS Y DISCUCIÓN}

\section{Deducción del modelo}

En la ecuación (2) se definió el pH operacional como el modelo básico para medir el $\mathrm{pH}$ de una solución problema $\left(\mathrm{pH}_{\mathrm{x}}\right)$. Si $\mathrm{E}_{\mathrm{B}}$ y $\mathrm{E}_{\mathrm{X}}$ se miden en $\mathrm{mV}$, se puede designar la constante $A=2303(R / F)$. En estas condiciones la ecuación (2) se puede escribir como (3).

$$
p H_{X}=p H_{B}+\frac{\left(E_{B}-E_{X}\right)}{A T}
$$

El $\mathrm{pH}_{\mathrm{B}}$ (7.00) debe corresponder teóricamente al $\mathrm{pH}$ donde el potencial $E_{B}$ es $0 \mathrm{mV}$ y no depende de la temperatura. No obstante, ningún electrodo cumple con estas condiciones debido a las imperfecciones de la membrana de vidrio durante su fabricación, estableciéndose un potencial de asimetría que equivale a la diferencia de potencial entre el $\mathrm{pH}$ a $0.000 \mathrm{~V}\left(\mathrm{pH}_{0}\right.$ a la temperatura de calibración, $25^{\circ} \mathrm{C}$ ) y el $\mathrm{pH}$ en el punto donde el potencial es independiente de la temperatura denominado isopotencial $\left(\mathrm{pH}_{\text {iso }}\right)^{[2]}$. En estas condiciones, la ecuación (3) se puede expresar como:

$$
p H_{X}=p H_{\text {iso }}+\frac{A T_{25}\left(p H_{0}-p H_{\text {iso }}\right)-E_{X}}{A T_{25}}
$$

Si se considera la corrección de la pendiente $\mathrm{P}_{25}$ (relación entre la pendiente práctica con respecto a la teórica a $25^{\circ} \mathrm{C}$ ) y el factor de corrección de la temperatura debido a la diferencia de temperatura entre la solución de referencia $\left(T_{25}\right)$ y la muestra $\left(T_{x}\right)$, se tiene el modelo matemático buscado, ecuación (5).

$$
p H_{X}=p H_{\text {iso }}+\frac{P_{25} A T_{25}\left(p H_{0}-p H_{\text {iso }}\right)-E_{X}}{P_{25} A T_{X}}
$$

\section{Identificación de los componentes de la incertidumbre}

Existen 7 componentes principales que influyen en la incertidumbre en $\mathrm{pH}_{\mathrm{x}}$ : el potencial $\mathrm{E}_{\mathrm{x}}$ de la celda medido en la muestra de agua, el $\mathrm{pH}$ isopotencial donde no depende de la temperatura, el pH de calibración donde el potencial de la celda es nulo a $25^{\circ} \mathrm{C}$, la temperatura medida en la muestra, la temperatura de calibración $\left(25^{\circ} \mathrm{C}\right)$, la constante $A$ como función de las incertidumbres en los valores de $R$ y $F$, el factor de corrección de la pendiente a $25^{\circ} \mathrm{C}$.

Cada uno de estos componentes principales tiene otras fuentes de incertidumbre, los cuales se detallan en el diagrama causa-efecto ${ }^{[13]}$ que se presenta en la figura 2. 


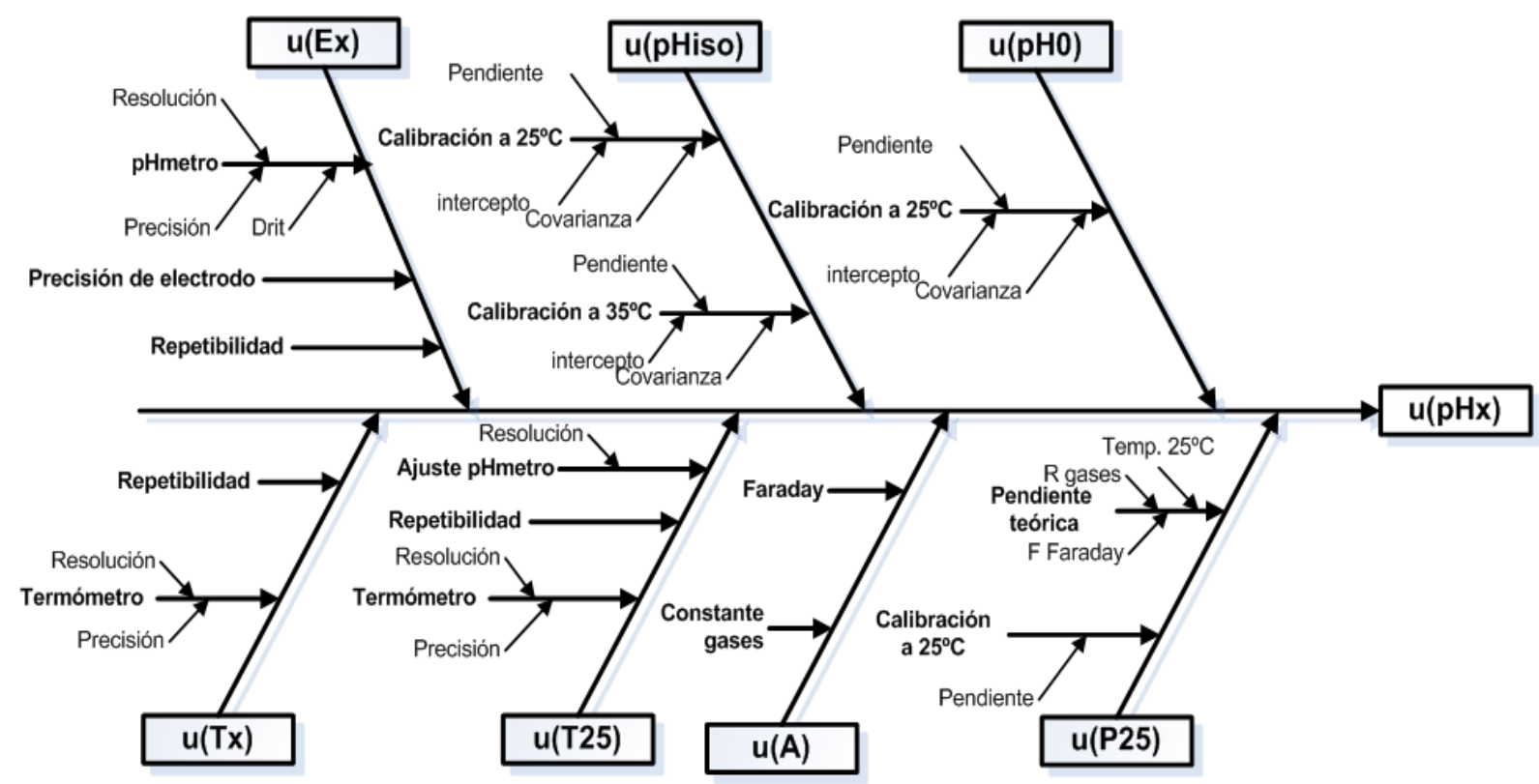

Figura 2. Diagrama causa-efecto para los componentes de la incertidumbre en la medición del pH en una muestra de agua.

\section{Cuantificación de los componentes de la incertidumbre}

\section{Cálculo de la incertidumbre del $\mathrm{pH}_{\text {iso }}$}

Tal a como se afirmó anteriormente, el $p H_{i s o}$ es el valor de $p H$ donde el potencial es independiente de la temperatura. Es el eje de rotación o centro de gravedad de la recta que representa el potencial de la celda como función del $p H$. Para evaluarlo fue necesario medir el potencial de tres soluciones de referencia ( $p H$ : 4.00, 7.00 y 10.00 trazables con NIST) a dos diferentes temperaturas $\left(25^{\circ} \mathrm{C}\right.$ y $35^{\circ} \mathrm{C}$ para el presente estudio) y luego se aplicó un análisis de regresión lineal con el fin de obtener las rectas y evaluar su intersección a través de la ecuación (6). Los resultados se presentan en las tablas 1 y 2 ; la intersección de las dos rectas se puede ver en la figura 3 .

TABLA 1. Valores de potencial (Ei) en $m V$ a diferentes $p H$ de soluciones de referencia medidos en réplicas de 5 a 25 y $35^{\circ} \mathrm{C}$

\begin{tabular}{|l|c|c|c|c|c|c|}
\hline$T^{\circ} C$ & $p H$ & $E_{1}$ & $E_{2}$ & $E_{3}$ & $E_{4}$ & $E_{5}$ \\
\hline \multirow{3}{*}{25} & 4 & 166 & 168 & 168 & 168 & 167 \\
\cline { 2 - 7 } & 7 & -7 & -6 & -6 & -7 & -6 \\
\cline { 2 - 7 } & 10 & -182 & -180 & -180 & -183 & -182 \\
\hline \multirow{3}{*}{35} & 4 & 178 & 178 & 179 & 179 & 177 \\
\cline { 2 - 7 } & 7 & -3 & -3 & -2 & -3 & -2 \\
\cline { 2 - 7 } & 10 & -190 & -188 & -187 & -188 & -188 \\
\hline
\end{tabular}

$$
p H_{\text {iso }}=\frac{{ }^{25} b_{0}-{ }^{35} b_{0}}{{ }^{35} b_{1}-{ }^{25} b_{1}}(6)
$$

El valor de pHiso es 8.92, calculado a partir de la ecuación (6), la cual fue obtenida por la igualdad de los dos modelos de calibración (a 25 y $35^{\circ} \mathrm{C}$ ) y el potencial en este punto es $-119 \mathrm{mV}$. Este valor de potencial corresponde al potencial de asimetría del electrodo utilizado en este estudio. Para calcular la incertidumbre en $p H_{i s o}$, se aplicó la ley de a propagación de la incertidumbre ${ }^{[12]}$ de acuerdo a la ecuación (7).

Es importante remarcar que los coeficientes del modelo de regresión $\left(b_{0} \mathrm{y} b_{1}\right)$ son parámetros correlacionados ${ }^{[23]}$, por tanto se debe calcular la covarianza, la cual está representada en el segundo término de la ecuación (7). En anexo se puede ver la derivación de esta ecuación.

$$
u p H_{i s o}=\sqrt{\sum c_{b i}^{2} u_{b i}^{2}+2 \sum \sum c_{b i} c_{b j} u_{b i} u_{b j} r\left(b_{i}, b_{j}\right)}(7)
$$

Donde $c_{b i}$ son los coeficientes de sensibilidad para los interceptos $(i=0)$ y las pendientes $(i=1)$ a 25 y $35^{\circ} \mathrm{C}$ calculadas a partir de las derivadas parciales de pHiso con respecto a $b_{i}, c_{b i}=\partial p H_{i s o} / \partial b_{i}$; en el segundo término, $c_{b i}$ y $c_{b j}$ son los coeficientes de sensibilidad de los interceptos $(i=0)$ y las pendientes $(j=1)$ a 25 y 35 ${ }^{\circ} \mathrm{C} ; u_{b i}$ son las incertidumbres de los interceptos y las pendientes calculadas por $S_{b_{l}} / \sqrt{ } 15 ; r\left(b_{\mathrm{i}}, b_{\mathrm{j}}\right)$ es el coeficiente de correlación entre $b_{0}$ y $b_{1}$ a 25 y $35^{\circ} \mathrm{C}$. Los datos necesarios para el cálculo de $u p H_{i s o}$ se encuentran en la tabla $\mathrm{N}^{\circ} 3$. 


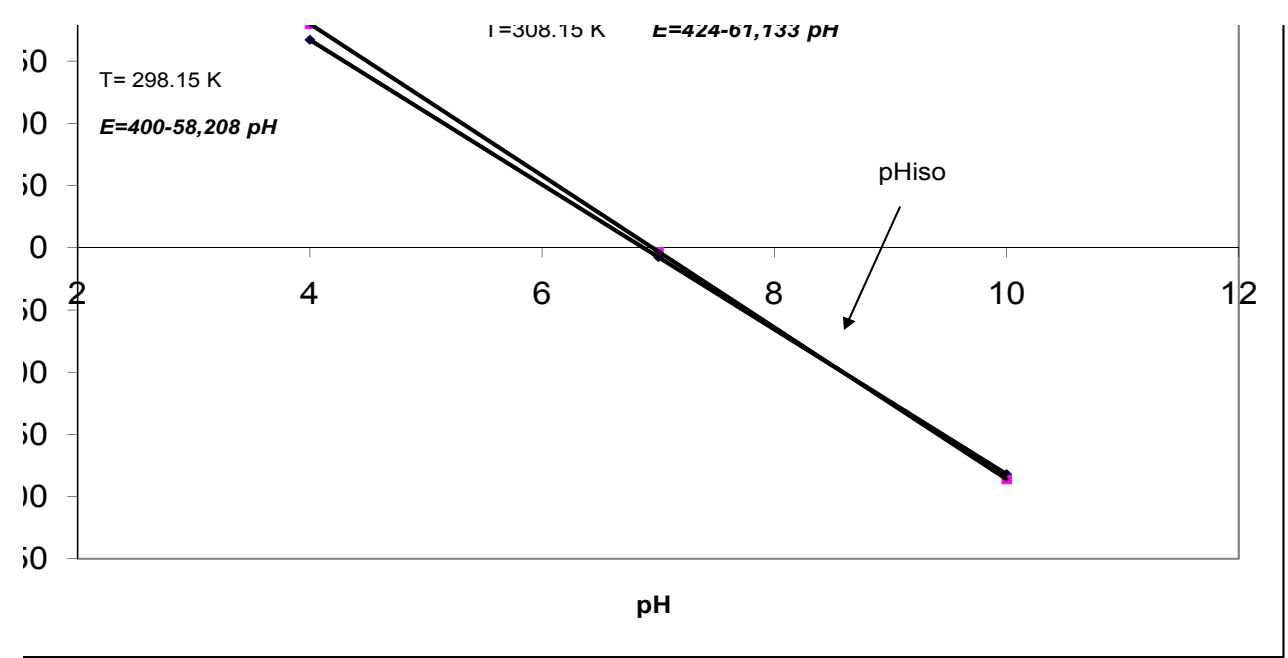

Figura 3. Modelos de calibración de potencial en función del pH a 25 y $35^{\circ} \mathrm{C}$. El valor de pHiso es de 8.92.

TABLA 2. Resultados del análisis de regresión lineal a 25 y $35^{\circ} \mathrm{C}$

\begin{tabular}{|l|c|c|c|c|c|}
\hline$T /{ }^{\circ} \mathrm{C}$ & $\begin{array}{c}\text { Pendiente } \\
b_{1}\end{array}$ & $S_{b 1}$ & $\begin{array}{c}\text { Intercepto } \\
b_{0}\end{array}$ & $S_{b 0}$ & $r^{2}$ \\
\hline 25 & -58.208 & 0.15146 & 400 & 1.0007 & 0.9999 \\
\hline 35 & -60.898 & 0.18691 & 422 & 1.4315 & 0.9999 \\
\hline
\end{tabular}

TABLA 3. Datos para el cálculo (*) de upHiso.

\begin{tabular}{|c|c|c|c|c|}
\hline Parámetro & $b_{i}$ & $u_{b i}$ & $c_{b i}$ & $\left(c_{b i}{ }^{*} u_{b i}\right)^{2}$ \\
\hline${ }^{25} \mathrm{~b}_{0}$ & 400 & 0.25837962890 & -0.3717472119 & 0.00922596878 \\
\hline${ }^{25} \mathrm{~b}_{1}$ & -58.208 & 0.03910680383 & -3.3167037490 & 0.01682356418 \\
\hline${ }^{35} \mathrm{~b}_{\mathrm{i}}$ & 424 & 0.36961171060 & 0.3717472190 & 0.01887934340 \\
\hline${ }^{35} \mathrm{~b}_{\mathrm{i}}$ & -60.898 & 0.04825995449 & 3.3167037490 & 0.02562047514 \\
\hline${ }^{25} \mathrm{r}\left(\mathrm{b}_{0} \mathrm{~b}_{1}\right)$ & -0.9613 & \\
\hline${ }^{35}\left(\mathrm{~b}_{0} \mathrm{~b}_{1}\right)$ & -0.9621 & \\
\hline
\end{tabular}

(*) Para una mayor precisión los cálculos fueron realizados con el programa de cálculo formal MAPLE 11 .

Si se sustituyen estos datos en la ecuación (7), se tiene la estimación de la incertidumbre de pHiso:

$$
u p H_{\text {iso }}=\sqrt{\begin{array}{l}
0.00922596878+0.01682356418+0.01887934+0.02562047514+ \\
2(-0.3717472119)(-3.316703749)(0.2583796289)(0.03910680383)(-0.9613)+
\end{array}}=0.0654
$$

Los grados de libertad se pueden calcular a partir del conjunto de 15 mediciones efectuadas para estimar los dos modelos regresión: $v_{\text {pHiso }}=2\left(\mathrm{n}_{1}-2\right)=26$.

\section{Cálculo de la incertidumbre de $\mathrm{pH}_{0}$ a la temperatura de calibración $25^{\circ} \mathrm{C}$}

El valor de $\mathrm{pH}$ cuando el potencial es $0.000 \mathrm{~V}\left(\mathrm{pH}_{0}\right)$ se obtiene a partir del modelo de calibración a $25^{\circ} \mathrm{C}$ $(E=400-58.208 \mathrm{pH})$. Los coeficientes del modelo se encuentran en la tabla 2 y la expresión matemática para el cálculo de $\mathrm{pH}_{0}$ es la siguiente:

$$
p H_{0}=\frac{b_{0}}{b_{1}}=6.87
$$

Para calcular la incertidumbre de $\mathrm{pH}_{0}$ se aplica la ley de propagación de la incertidumbre a la ecuación (8). La expresión para calcular la incertidumbre de $\mathrm{pH}_{0}$ se representa en la ecuación (9).

$$
u p H_{0}=\sqrt{c_{b 0}^{2} u_{b 0}^{2}+c_{b 1}^{2} u_{b 1}^{2}+2 c_{b 0} c_{b 1} u_{b 0} u_{b 1} r\left(b_{0}, b_{1}\right)}
$$

Tomando los datos necesarios en la tabla $\mathrm{N}^{\circ} 3 \mathrm{e}$ introduciéndolos en la ecuación anterior se tiene el valor de la incertidumbre en $\mathrm{pH}_{0}$.

$$
u_{p H O}=0.0458
$$

Los grados de libertad se obtienen a partir de las 15 mediciones realizadas para estimar el modelo de regresión: $v=\mathrm{n}-2=13$

\section{Cálculo de la incertidumbre de $A$}

La expresión matemática de $\mathrm{A}$ en $\mathrm{mV} \mathrm{pH}^{-1} \mathrm{~K}^{-1}$ es la siguiente:

$$
A=2303 \frac{R}{F}
$$

Admitiendo que las variables $R$ y $F$ no están correlacionadas y aplicando la ley de la propagación de la incertidumbre se tiene la ecuación (11).

$$
u A=\sqrt{c_{R}^{2} u_{R}^{2}+c_{F}^{2} u_{l}^{\prime}}(11)
$$

Los valores de $\mathrm{R}$ y $\mathrm{F}$ son $8.31441 \mathrm{~J} \mathrm{~mol}^{-1} \mathrm{~K}^{-1} \mathrm{y}$ $96484.56 \mathrm{Cmol}^{-1}$. Considerando 10 mediciones, las incertidumbres estándares de $\mathrm{R}$ y $\mathrm{F}$ son $u_{R}=0.00026 / \sqrt{ } 10$ 
y $u_{F}=0.52 / \sqrt{ } 10{ }^{[18]}$. Los coeficientes de sensibilidad calculados por la derivada parcial de la función A con respecto a $R$ y $F$ son: $c_{R}=0.0239$ y $c_{F}=-0.000206$. Sustituyendo los datos en (10) y (11) se tienen el valor de A y su incertidumbre.

$$
\begin{gathered}
\mathrm{A}=0.198444 \mathrm{mVpH}^{-1} \mathrm{k}^{-1} \\
u_{\mathrm{A}}=0.000199
\end{gathered}
$$

Los grados de libertad en la estimación de la incertidumbre de A se calculan a partir de las dos series de 10 mediciones en $\mathrm{R}$ y $\mathrm{F}: v=2(\mathrm{n}-1)=18$

\section{Incertidumbre en la temperatura de calibración $T_{25}$}

La incertidumbre en la temperatura de calibración a $25{ }^{\circ} \mathrm{C}\left(u_{T 25}\right)$ tiene dos componentes: la incertidumbre del termómetro y la del ajuste de la temperatura en el pHmetro. El detalle de los cálculos de $u_{T 25}$ se encuentran en la tabla $\mathrm{N}^{\circ} 4$. Los grados de libertad efectivos $\left(v_{\mathrm{ef}}\right)$ calculados según la ecuación (7) tienen un valor de 140.

TABLA 4.Componentes de la incertidumbre de la temperatura de

\begin{tabular}{|c|c|c|c|c|c|}
\hline Instrumento & Fuente & $\begin{array}{l}\text { Especificación } \\
\text { (a) en }{ }^{\circ} C^{\prime}(\text { ) }\end{array}$ & Distribución & $\begin{array}{l}\text { Fórmula y } \\
\text { grados de } \\
\text { libertad (v) }\end{array}$ & $\boldsymbol{u}_{i}$ \\
\hline \multirow[t]{3}{*}{ Termómetro } & Resolución & 0.1 & Rectangular & $\begin{array}{l}u_{R 1}=\frac{a}{2 \sqrt{3}} \\
v=\infty\end{array}$ & 0.0288 \\
\hline & $\begin{array}{l}\text { Incertidumbre } \\
\text { según } \\
\text { fabricante }\end{array}$ & \pm 0.1 & Normal & $\begin{array}{l}u_{f}=\frac{a}{1.96} \\
v=50\end{array}$ & 0.051 \\
\hline & $\begin{array}{l}\text { Repetibilidad } \\
\left(S_{r}\right)\end{array}$ & $0.07^{(* *)}$ & t Student & $\begin{array}{l}u_{r}=\frac{S_{r}}{\sqrt{n}} \\
v=9\end{array}$ & 0.02134 \\
\hline pHmetro & Resolución & 0.1 & Rectangular & $\begin{array}{l}u_{R 2}=\frac{a}{2 \sqrt{3}} \\
\nu=\infty\end{array}$ & 0.0288 \\
\hline
\end{tabular}
calibración a $25^{\circ} \mathrm{C} T_{25}$.

(*) Las especificaciones son obtenidas de los catálogos del termómetro y del pHmetro. $\left(^{* *}\right)$ Valor obtenido a partir de una serie de 10 mediciones. El promedio de $T_{25}$ es $298.02 \mathrm{~K}$.

\section{Cálculo de la incertidumbre de $\boldsymbol{P}_{25}$}

El factor de corrección de la pendiente a $25^{\circ} \mathrm{C}$ está dado por la ecuación (12). Es la relación entre la pendiente experimental $\left(b_{\text {lexp }}\right)$, obtenida a partir del modelo de calibración del potencial medido para los valores de $\mathrm{pH}$ de las soluciones de referencias certificadas, y la pendiente teórica $\left(A T_{25}=59.16 \mathrm{mV}\right)$.

$$
P_{25}=\frac{b_{1 \exp }}{A T_{25}}
$$

Dada que, las dos pendientes son variables independientes, la incertidumbre en $\mathrm{P}_{25}$ se calcula, según la siguiente ecuación:

$$
u P_{25}=\sqrt{c_{b 1 \exp }^{2} u_{b 1 \exp }^{2}+c_{A}^{2} u_{A}^{2}+c_{T 25}^{2} u_{T 25}^{2}}(13)
$$

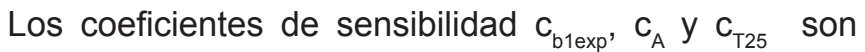
$0.016909,-4.959753, y-0.00330$. Las incertidumbres estándares $u_{\text {blexp }}, u_{A}$ y $u_{T 25}$ tienen valores de 0.03911, 0.000199 y 0.06867 respectivamente. La incertidumbre de $b_{1 \text { exp }}$ se obtiene a partir de la desviación estándar de la recta de calibración a $25^{\circ} \mathrm{C}$ (ver tabla 2) para 15 mediciones. Sustituyendo estos datos en (13) se tiene la incertidumbre en $\mathrm{P}_{25}$, cuyo valor es el siguiente:

$$
u_{\mathrm{P} 25}=0.00187
$$

El valor de $\mathrm{P}_{25}$ es:

$$
P_{25}=58.208 /(0.198444 \times 298.02)=0.9842
$$

Los grados de libertad obtenidos a partir de la ecuación (17) tienen un valor de 31 grados de libertad efectivos.

\section{Cálculo de la incertidumbre de la temperatura medida en una muestra de agua $u_{T X}$}

Las fuentes que afectan la incertidumbre en la medición de la temperatura en la muestra de agua son tres: la debida a la resolución de la lectura del instrumento, la de la precisión especificada por el fabricante en el catálogo del termómetro ${ }^{[25]}$ y la debida a la repetibilidad. Las dos primeras se encuentran en la tabla 4 (0.0288 y 0.051 respectivamente).

La incertidumbre de repetibilidad se obtuvo a partir de la desviación estándar de 10 mediciones de la temperatura en la muestra de agua: $\mathrm{t}^{\circ} \mathrm{C}: 28.1,27.8,27.9,27.8,27.9$, $28.0,28.0,27.9,27.8,28.0$.

El promedio y la desviación estándar en ${ }^{\circ} \mathrm{C}$ son 27.92 y 0.103 respectivamente. El valor de la incertidumbre estándar de repetibilidad es $0.103 / \sqrt{ } 10$, igual a 0.0326 . Por tanto la incertidumbre de la medición de la temperatura en la muestra de agua y el valor de Tx en unidades $\mathrm{K}$ son:

$$
\begin{gathered}
u T x=\sqrt{ }(0.02882+0.0512+0.03262)=0.0670 \mathrm{~K} \\
T x=273.15+27.9=301.07 \mathrm{~K}
\end{gathered}
$$

Los grados de libertad efectivos en la medición de uTx son 77 .

Cálculo de la incertidumbre en la medición del potencial en una muestra de agua $u_{E X}$

La incertidumbre en la medición del potencial de la muestra de agua $E_{x}$, tiene dos componentes, la del 
instrumento potenciométrico ( $\mathrm{pHmetro}+$ electrodo) y la de repetibilidad. En la tabla 5 , se presenta el cálculo de los diferentes componentes de la incertidumbre en el potencial $E_{x}$. La resolución del pHmetro y la incertidumbre expandida reportada por el fabricante son de $1 \mathrm{mV}$ y $\pm 1 \mathrm{mV}$ respectivamente.

TABLA No 5. Datos y cálculos para la evaluación de la incertidumbre de $u_{E X}$

\begin{tabular}{|c|c|c|c|c|c|}
\hline Instrumento & Fuente & $\begin{array}{l}\text { Especificación } \\
\text { (a) }\end{array}$ & Distribución & \begin{tabular}{|l|} 
Fórmula \\
grados \\
libertad
\end{tabular} & $u_{i}$ \\
\hline \multirow[t]{3}{*}{ pHmetro } & Resolución & $1 \mathrm{mv}$ & Rectangular & $\begin{array}{c}u_{R}=\frac{a}{2 \sqrt{3}} \\
v=\infty\end{array}$ & 0.289 \\
\hline & Precisión & $+/-1 \mathrm{mv}$ & Normal & $\begin{array}{c}u_{r}=\frac{a}{1.96} \\
v=50\end{array}$ & 0.510 \\
\hline & Drift en $12 \mathrm{~h}$ & $<3 \mathrm{mv}$ & Rectangular & $\begin{array}{c}u_{D}=\frac{a}{2 \sqrt{3}} \\
v=\infty\end{array}$ & 0.866 \\
\hline \multirow[t]{2}{*}{ Electrodo } & $\begin{array}{l}\text { Incertidumbre } \\
\text { del electrodo }\end{array}$ & $+/-1 \mathrm{mv}$ & Normal & $\begin{aligned} u_{e l} & =\frac{a}{1.96} \\
v & =50\end{aligned}$ & 0.510 \\
\hline & $\begin{array}{c}\text { Desviación } \\
\text { estándar de } \\
\text { repetibilidad }(S r)\end{array}$ & $1.986 \mathrm{mV}$ (") & $\mathrm{t}$ de Student & $\begin{array}{c}u_{r}=\frac{S_{r}}{\sqrt{9}} \\
\mathrm{v}=8\end{array}$ & 0.662 \\
\hline
\end{tabular}

(*) Obtenido a partir de 9 mediciones de potencial en $m V$ de la muestra de agua: $E_{X i}:-46,-41,-42,-42,-40,-43,-41,-39,-42$.

El Drift o variación del potencial en $12 \mathrm{~h}$ es $<3 \mathrm{mV}$ [2]. El electrodo presenta una incertidumbre de $\pm 1 \mathrm{mV}$. La incertidumbre debido a la repetibilidad se obtiene a partir de 10 mediciones de potencial a la muestra de agua. La incertidumbre $u_{E x}$ se calcula según la ecuación (14).

$$
u_{E x}=\sqrt{\sum u_{i}^{2}}(14)
$$

Sustituyendo los valores de $u_{i}$ de la tabla 5 , se tiene:

$$
u_{E x}=1.339 \mathrm{mV}
$$

Los grados de libertad efectivos tienen un valor estimado en 120. El potencial promedio medido en la muestra de agua es:

$$
E x=-41.77 \mathrm{mV}
$$

\section{Balance de las incertidumbres}

En la tabla 6, se resumen todos los parámetros que intervienen en la evaluación de la incertidumbre de $\mathrm{pH} x$. En esta tabla se muestran las variables $\left(x_{i}\right)$ del modelo matemático de la ecuación (5), el valor correspondiente, las incertidumbres $\left(u_{x i}\right)$, los coeficientes de sensibilidad de cada variable $\left(\mathrm{c}_{\mathrm{xi}}\right)$ calculados a partir de la derivada parcial de $p H x$ con respecto a $x_{i} \quad\left(c_{x i}=\frac{\partial p H_{x}}{\partial x_{i}}\right.$, el producto de $c_{x i}$ y $u_{x i}$, y el índice de contribución (Ind\%) calculado a partir de la ecuación (15).

$$
\operatorname{Ind} \%=\frac{\left(c_{x i} u_{x i}\right)^{2}}{\sum\left(c_{x i} u_{x i}\right)^{2}}(100
$$

Si se realiza un diagrama de barra para Ind\% en función de los parámetros y las sumas acumuladas se obtiene el Diagrama de Pareto ${ }^{[22]}$.

La figura 4, representa este diagrama, donde se puede observar que los parámetros $\mathrm{pH}_{0}$ y Ex son los que mayormente contribuyen (más del 90\%) a la incertidumbre en la medición de $\mathrm{pH}$ en la muestra de agua.

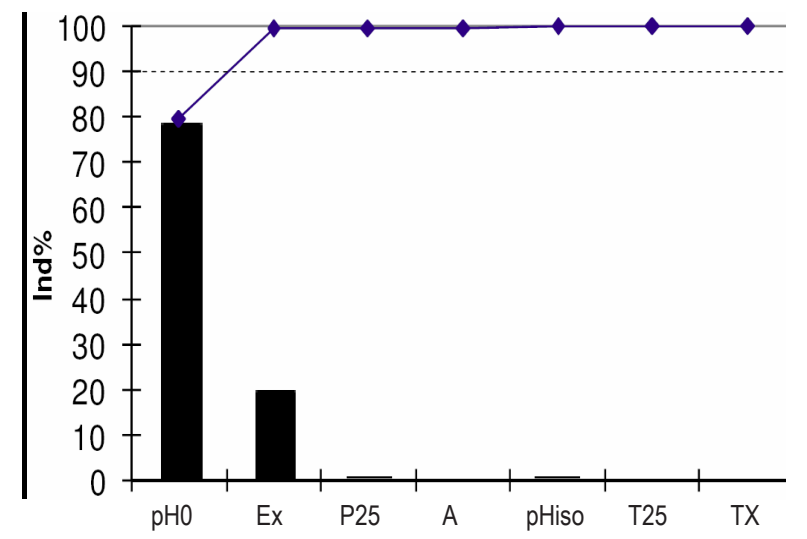

\begin{tabular}{|c|c|c|c|c|c|}
\hline Parámetro $\left(x_{i}\right)$ & Valor de $\left(x_{i}\right)$ & $\mu_{x i}$ & $c_{x i}$ & $\left(c_{x i} \mu_{x i}\right)^{2}$ & Ind $\%$ \\
\hline$p H_{i s o}$ & 8.92 & 0.0654 & 0.01013 & $4.3896 \times 10^{-6}$ & 0.2 \\
\hline$p H_{0}$ & 6.87 & 0.0458 & 0.98989 & 0.002055 & 79.6 \\
\hline$P_{25}$ & 0.9842 & 0.00187 & -0.72176 & $1.822 \times 10^{-6}$ & 0.1 \\
\hline$T_{25}$ en $K$ & 298.02 & 0.06867 & -0.006809 & $2.186 \times 10^{-7}$ & 0.0 \\
\hline$T_{X}$ en $K$ & 301.07 & 0.0670 & 0.00438 & $8.614 \times 10^{-8}$ & 0.0 \\
\hline A en $m V K^{-1} p H^{-1}$ & 0.198444 & 0.000199 & -3.5796 & $5.074 \times 10^{-7}$ & 0.0 \\
\hline$E_{X}$ en $m V$ & -41.77 & 1.339 & -0.017006 & $5.185 \times 10^{-4}$ & 20.1 \\
\hline & & & Suma= & 0.00258 & 100 \\
\hline
\end{tabular}

Figura 4. Diagrama de Pareto para las contribuciones de las incertidumbres en $\mathrm{upH}_{x}$.

TABLA No 6. Balance de la incertidumbre en la medición del pH en una muestra de agua. 
Si se asume, que la influencia es considerable tomando como referencia el $90 \%$, se puede ver que, basta tomar en cuenta solamente los componentes de las incertidumbres del $\mathrm{pH}_{0}$ y $\mathrm{E}_{\mathrm{x}}$ para estimar la incertidumbre total de $\mathrm{pHx}$.

\section{Estimación de la incertidumbre combinada en la medición de $\mathrm{pH}$ en la muestra de agua $u \mathrm{pH}_{\mathrm{x}}$}

Aplicando la ley de propagación de la incertidumbre al modelo matemático representado en la ecuación (5), se tiene la ecuación (16).

$$
u p H_{x}=\sqrt{c_{p H H i o}^{2} u_{p H H o O}^{2}+c_{p H}^{2} u_{p H 0}^{2}+c_{P 25}^{2} u_{P 25}^{2}+c_{A}^{2} u_{A}^{2}+c_{T 25}^{2} u_{T 25}^{2}+c_{T x}^{2} u_{T x}^{2}+c_{E x}^{2} u_{E x}^{2}}
$$

Si sustituimos los datos de la quinta columna de la tabla $N^{\circ} 6$ en la ecuación anterior, se tiene el valor de la incertidumbre combinada de la medición de $\mathrm{pH}$ en la muestra de agua.

$$
u_{p H x}=0.05
$$

Este mismo resultado se obtiene, si se sustituyen en la ecuación (16) solamente los términos correspondientes a los parámetros $\mathrm{E}_{\mathrm{x}}$ y $\mathrm{pH}_{0}$.

\section{Estimación del intervalo de confianza}

Para evaluar la incertidumbre expandida, es necesario seleccionar el factor de cobertura $k$. Este factor puede ser seleccionado de acuerdo al tipo de distribución. Así, para una distribución normal y el $95.5 \%$ de nivel de confianza $k=2$; para una distribución $\mathrm{t}$ se pueden seleccionar dos valores: $k=t_{0.975, v} 0 t_{0.975, v / N n}$.

Generalmente, cuando se reporta la incertidumbre expandida, se selecciona el valor de 2 asumiendo que los resultados siguen una distribución normal al 95.5\% de nivel de confianza. No obstante, cualquier valor de $k$ seleccionado se debe declarar cuando se reportan las incertidumbres expandidas.

Una forma rigurosa de seleccionar $k$ es evaluando los grados de libertad efectivos $\left(v_{\mathrm{ef}}\right)$ según la ecuación (17) ${ }^{[9,12]}$. Si este valor es mayor o igual a 30 se selecciona el factor $k=2$; si es menor de $30, k$ debe seleccionarse de acuerdo a la distribución $t$.

$$
v_{e f}=\frac{u_{p H x}^{4}}{\sum \frac{c_{i}^{4} u_{x i}^{4}}{v_{i}}}
$$

Los datos necesarios para calcular $v_{e f}$ se encuentran en la tabla 7. En esta tabla se encuentran los grados de libertad intermediarios vi para cada uno de los componentes de la incertidumbre que ya fueron evaluados anteriormente. Al dividir la incertidumbre de
$\mathrm{pH}_{\mathrm{x}}$ elevada a la cuarta potencia $\left(u p H^{4}\right)$ entre la suma de la última columna de la tabla anterior se obtienen los grados de libertad efectivos. El valor calculado de esta manera es el siguiente: $v_{\mathrm{ef}}=18$

TABLA 7. Datos para el cálculo de los grados de libertad efectivos $v_{\text {er }}$

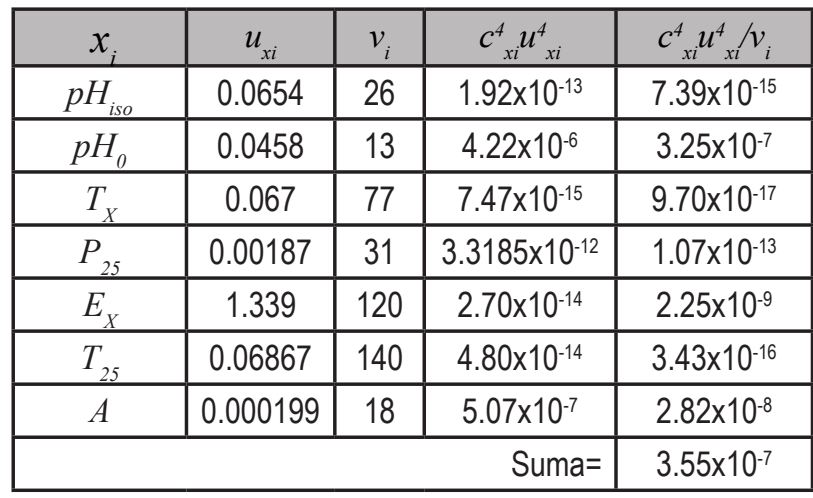

Para evaluar la incertidumbre expandida el factor $k$ es el valor de $t$ de las tablas de distribución $\mathrm{t}$ a dos colas y al $95 \%$ de nivel de confianza. Este valor es 2.101. La incertidumbre expandida se expresa de la siguiente manera:

$$
U=t_{0.975,18} u p H_{X}=2.101 \times 0.05=0.11
$$

El intervalo de confianza del $\mathrm{pH}$ medido en la muestra de agua es:

$$
p H_{X} \pm U=7.60 \pm 0.11
$$

La precisión en la medición del pH en la muestra de agua, puede evaluarse como la incertidumbre expandida relativa al $p H x$ expresada en porcentaje (UER\%). En la práctica, dependiendo de la calidad del electrodo y de la precisión del pHmetro, se pueden tener valores de incertidumbres expandidas de hasta 0.1 tomando un factor de cobertura $k=2$.

Si se grafica UER\% en la gama de $\mathrm{pH}$ entre 2 y 11 para $0.5 \%$ y $1 \%$, se observa que, las incertidumbres disminuyen cuando aumenta el valor de $\mathrm{pH}$. En la figura 5 , se observa este fenómeno. En el presente estudio el valor de UER\% es de $\pm 1.41 \%$. Estos puntos se observan en la gráfica y puede verse que están en los límites aceptables.

Si se mide directamente el $p H_{x}$ de la muestra cuya temperatura promedio fue evaluada anteriormente $\left(27.9^{\circ} \mathrm{C}\right)$ se tienen los siguientes resultados: $7.79,7.71$, 7.71, 7.72, 7.68, 7.73, 7.71, 7.66, 7.71. Con un valor promedio de 7.71 y una desviación estándar igual a 0.03 . 


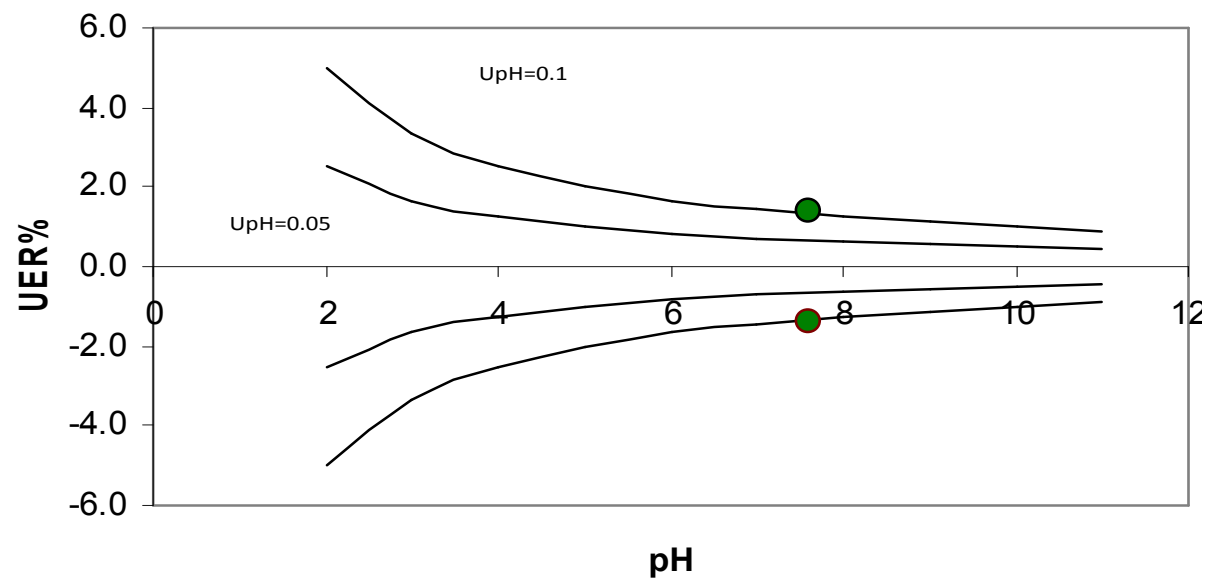

Figura 5. Incertidumbre expandida relativa UER\% en las mediciones de $\mathrm{pH}$ obtenidas con dos electrodos de diferentes precisiones. Los círculos marcados representan los resultados experimentales del presente estudio (UER\%₫1.41).

Al evaluar el intervalo de confianza utilizando solamente la desviación estándar de repetibilidad y 8 grados de libertad se tiene:

$$
\begin{aligned}
& p H x \pm t_{0.975,8} s_{x} \\
& 7.71 \pm(2.31)(0.03) \\
& 7.71 \pm 0.07
\end{aligned}
$$

Si se comparan los dos intervalos (18) y (19), se observa que no existen diferencias significativas, lo que confirma la calidad en las mediciones del $\mathrm{pH}$ en la muestra de agua. Sin embargo, el método riguroso utilizado en este estudio demuestra que no solamente la repetibilidad influye en la incertidumbre, si no que existen otros parámetros experimentales que deben ser tomados en cuenta en el cálculo. El parámetro que más influye en esta evaluación, es el $\mathrm{pH}$, donde el potencial es nulo $\left(u p H_{0}\right)$ a la temperatura de calibración $\left(25^{\circ} \mathrm{C}\right)$.

\section{CONCLUSIONES}

La metodología desarrollada en el presente trabajo, estableció un procedimiento riguroso para evaluar la incertidumbre en la medición del $\mathrm{pH}$ en una muestra de agua, basándose en la guía GUM ISO 1995. En primer lugar se dedujo el modelo matemático, partiendo de la definición de $\mathrm{pH}$ operacional y evaluando los valores de $\mathrm{pH}$ donde el potencial es independiente de la temperatura ( $p H i s o$ ) y donde el potencial es nulo a la temperatura de calibración a $25^{\circ} \mathrm{C}\left(\mathrm{pH}_{0}\right)$.

Seguidamente, se identificaron las fuentes de incertidumbres, a través del diagrama causa-efecto y se evaluaron cada una de los componentes de las incertidumbres, aplicando la ley de la propagación de la incertidumbre.
Por último, se aplicó el modelo matemático para determinar el $\mathrm{pH}$ en una muestra de agua de la red de distribución urbana y se estimó el intervalo de confianza utilizando la distribución t para 18 grados de libertad efectivos y $95 \%$ de nivel de confianza. La incertidumbre expandida relativa al $p H x$ UER\% es de 1.41 , lo que representa un valor experimental aceptable en el presente trabajo.

Un aspecto a destacar es que, de acuerdo al Diagrama de Pareto representado en la figura 4, los parámetros que contribuyen en más del 90 por ciento a la incertidumbre total de la medición de $p H x$, son el $p H_{0}$ de calibración y el potencial Ex medido en la muestra de agua. De estos dos parámetros experimentales, el primero tiene mayor influencia; esto significa que si se reduce la incertidumbre en $p H_{0}$, se puede obtener una mejor precisión en la estimación del $p H x$.

Para ello es necesario utilizar un mayor número de soluciones de referencia en una gama de $\mathrm{pH}$ entre 2 y 10 para la calibración a $25^{\circ} \mathrm{C}$. En el presente estudio se utilizaron solamente tres soluciones de referencia $(\mathrm{pH}$ $4.00,7.00$ y 10.00) trazables a los estándares primarios del Instituto Nacional de Normalización y Tecnología de los Estados Unidos (NIST).

\section{BIBLIOGRAFÍA}

1. Delgado, M., Vanegas, M. y Delgado G. (2007), "Metrología Química I: Calibración de un pHmetro y control de calidad", UNIVERSITAS, Número 1, vol 1, pag.14-23, León, Nicaragua.

2. Westcott, C., (1978), pH Measurements, N.Y, Academic Press, p.172. 
3. Clesceri, L., Greenberg, A. y Eaton, A., (1998), Standard Methods for the Examination of Water and Wastewater, N.Y., American Publishing Health Association, 20th edition, p. 2-24, 2-26.

4. Midgley, D. and Torrance, K., (1978), Potentiometric Water Analysis, N.Y., John Wiley, p. 135, 147.

5. ISO/IEC 17025(ES), (2005), "Requisitos Generales para la Competencia de los Laboratorios de Ensayo y de Calibración", Norma Internacional, Ginebra, Segunda Edición.

6. ISO/IEC 17011(ES), (2004), "Evaluación de la conformidad - Requisitos generales para los organismos de acreditación que realizan la acreditación de organismos de evaluación de la conformidad", Norma Internacional, Ginebra.

7. Bievre, P. (2006), "On quality of a measurement result", Accred. Qual. Assur, 11, 597-598.

8. Eurachem/Citac Guide, (2003), "Treceability in Chemical Measurement", Guía de Referencia, 1ra. Edición, UK.

9. Kimothi, S.K., (2000), The Uncertainty of Measurements, ASQ Quality Press, Wisconsin , pag. 391.

10. a) Leito, I., Strauss, L., Koort, E, y Phil, V.,(2000) Accred. Qual. Assur. 7 (2000) 242-249; b) KOORT, E., (2006), "Estimation of potentiomentrically measured $\mathrm{pH}$, and pKa Values" (Tesis doctoral), Tartu University PRESS, Estonia.

11. Aguiar R. et al., (2004), Guía Técnica sobre Trazabilidad e Incertidumbre en las Mediciones Analíticas que Emplean la Técnica de Medición de pH, CENAM/ema, México.

12. BIPM, IEC, IFCC, IUPAC, OIML, (1995) Guide for to the Expression of Uncertainty in Measurement (GUM), ISO, Ginebra.

13. Euracherm/Citac GUIDE, (2000), Quantifying Uncertainty in Analytical Chmistry, 2da edición, UK.

14. Philips S.D., Eberthardt KR, y Parry B, (1993) "Guidelines for expressing the uncertainty of measurement results containing uncorrected bias". Journal of Research of National Institute of Standards and Technology, 102, 5, 577-585
15. IZASA , (1998), "Trazabilidad en la medida de $\mathrm{pH}^{\text {". }}$ Industria Farmacéutica Analítica y Biotecnología, 13, 49-51.

16. Strobel, H., (1989), Chemical Instrumentation, $3^{\mathrm{a}}$ edición, John Wiley, NY, pág 1030.

17. IUPAC (1998), Compendium of Analytical Nomenclature, $3^{\mathrm{a}}$ edición, Oxford, pag 3-11

18. Dean J. (1979) Lange's Handbook of chemistry, $12^{\mathrm{a}}$ edición, McGraw Hill, N.Y.

19. Bates, R. G., (1963), Determination of $\mathrm{pH}, 2 \mathrm{da}$. Edición, John Wiley, NY. Pag 365.

20. Skoog, D. A., Holler, F. J. y Nieman, T. A., (2005), Análisis Instrumental, Madrid, 5ta. Edición, McGraw Hill, p.650.

21. Riley T. y Tomlinson, C. (1987), Principles of Electroanalytical Methods, John Wiley, London, pág. 57.

22. Wodsworth, H. M., Stephens, K. y Godfrey, A. B., (2005), Métodos de Control de Calidad, México, Compañía Editorial Continental, 1ra. Edición, p. 359.

23. Mathias, O., (1999), Chemometrics, Weinhein, Alemania, Wiley-VCH, p. 186

\section{ANEXO}




\section{Derivación de la ley de propagación de la incertidumbre}

Sea la función $Y=f_{(x)}$

Por la expansión de Taylor, despreciando las derivadas mayores de $2^{0}$ orden, se tiene:

$$
Y=\frac{f\left(x^{\prime}\right)\left(x-x^{\prime}\right)}{o !}+\frac{f^{\prime}\left(x^{\prime}\right)\left(x-x^{\prime}\right)}{1 !}+\ldots .
$$

Si hay más de una variable la función $Y$ se convierte en:

La esperanza estadística es:

$$
Y=f\left(x^{\prime}, z^{\prime}\right)+f^{\prime}\left(x^{\prime}\right)\left(x-x^{\prime}\right)+f^{\prime}\left(z^{\prime}\right)\left(z-z^{\prime}\right)
$$

$$
\begin{gathered}
E\left(y-f\left(x^{\prime}, z^{\prime}\right)\right)^{2}=E\left(f^{\prime}\left(x^{\prime}\right)\left(x-x^{\prime}\right)+f^{\prime}\left(z^{\prime}\right)\left(z-z^{\prime}\right)\right)^{2} \\
E\left(y-f\left(x^{\prime}, y^{\prime}\right)\right)^{2}=E\left(f^{\prime 2}\left(x^{\prime}\right)\left(x-x^{\prime}\right)+2 f^{\prime}\left(x^{\prime}\right) f^{\prime}(z)+f\left(z^{\prime}\right)^{2}\left(z-z^{\prime}\right)^{2}\right) \\
E\left(y-f^{\prime}\left(x^{\prime}, z^{\prime}\right)\right)^{2}=E\left[\left(f^{\prime 2}\left(x^{\prime}\right)\left(x-x^{\prime}\right)^{2}+f^{\prime 2}\left(z^{\prime}\right)\left(z-z^{\prime}\right)^{2}+2 f^{\prime}\left(x^{\prime}\right) f^{\prime}\left(z^{\prime}\right)\left(x-x^{\prime}\right)\left(z-z^{\prime}\right)\right)\right]
\end{gathered}
$$

Sustituyendo los términos de la esperanza estadística, $\left(x-x^{\prime}\right)^{2}$ y $\left(z-z^{\prime}\right)^{2}$ se transforman en incertidumbres al cuadrado $\left(\boldsymbol{u}_{i}^{2}\right)$, y tomando $f^{\prime}$ como las derivadas parciales de la función $Y$ con respecto a $x$ o $z$ la ecuación se transforma a:

$$
u_{(y)}^{2}=\left(\frac{\partial f}{\partial x}\right)^{2} u_{x}^{2}+\left(\frac{\partial f}{\partial z}\right)^{2} u_{z}^{2}+2\left(\frac{\partial f}{\partial x}\right)\left(\frac{\partial f}{\partial z}\right) u_{(x, z)}
$$

La anterior ecuación se puede generalizar y se convierte en la ecuación que representa la ley de propagación de la incertidumbre:

$$
u_{(y)}^{2}=\sum\left(\frac{\partial f}{\partial x_{i}}\right)^{2} u_{x i}^{2}+2 \sum_{i=1}^{N-1} \sum_{j=i+1}^{N}\left(\frac{\partial f}{\partial x_{i}}\right)\left(\frac{\partial f}{\partial x_{j}}\right) u_{(x i, x j)}
$$

Donde las derivadas parciales se denominan coeficientes de sensibilidad $c_{x i}$ y el segundo término se conoce como la covarianza para variables correlacionadas. $u\left(x_{i}, x_{j}\right)$ se puede evaluar por las incertidumbres de $x_{i}, x_{j}$ y el índice de correlación de estas variables, utilizando la ecuación siguiente:

$$
u_{(x i, x j)}=u_{x i} u_{x j} r_{(x i, x j)}
$$

En el caso específico de los coeficientes del modelo de regresión, el intercepto y la pendiente tienen una correlación negativa[12]. En el caso del modelo de calibración del presente estudio el índice de correlación se calcula según la siguiente fórmula:

$$
r\left(b_{0} b_{1}\right)=-\frac{\sum w_{i} p H_{i}}{\left[\sum w_{i} \sum w_{i} p H_{i}^{2}\right]^{1 / 2}}
$$

Donde $w_{i}$ es el factor de ponderación, el cual es inversamente proporcional a la varianza del potencial correspondiente. 